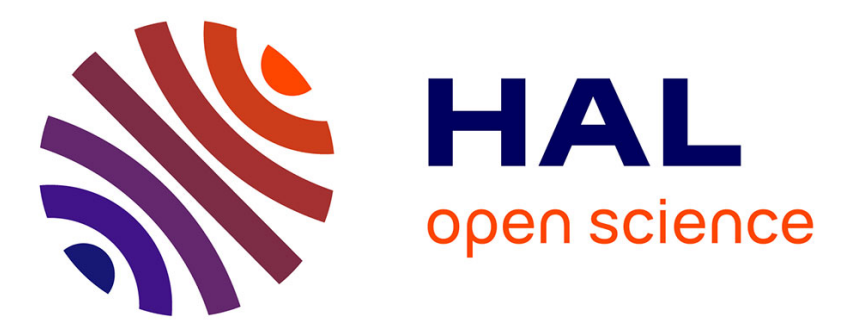

\title{
Detection of nanoparticles by single-particle ICP-MS with complete transport efficiency through direct nebulization at few-microlitres-per-minute uptake rates
}

\author{
Mickael Tharaud, Pascale Louvat, Marc F. Benedetti
}

\section{To cite this version:}

Mickael Tharaud, Pascale Louvat, Marc F. Benedetti. Detection of nanoparticles by single-particle ICP-MS with complete transport efficiency through direct nebulization at few-microlitres-per-minute uptake rates. Analytical and Bioanalytical Chemistry, 2020. hal-03024068

\author{
HAL Id: hal-03024068 \\ https://hal.science/hal-03024068
}

Submitted on 25 Nov 2020

HAL is a multi-disciplinary open access archive for the deposit and dissemination of scientific research documents, whether they are published or not. The documents may come from teaching and research institutions in France or abroad, or from public or private research centers.
L'archive ouverte pluridisciplinaire HAL, est destinée au dépôt et à la diffusion de documents scientifiques de niveau recherche, publiés ou non, émanant des établissements d'enseignement et de recherche français ou étrangers, des laboratoires publics ou privés. 


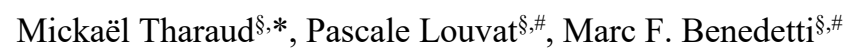

§Université de Paris, Institut de physique du globe de Paris, CNRS, F-75005 Paris, France

"Laboratoire Cogitamus, $13 / 4$ rue Descartes, 75005 Paris

\section{Corresponding author}

*e-mail: tharaud@ipgp.fr

tel.: +33-1-83-95-78-78

\section{Graphical abstract}

14

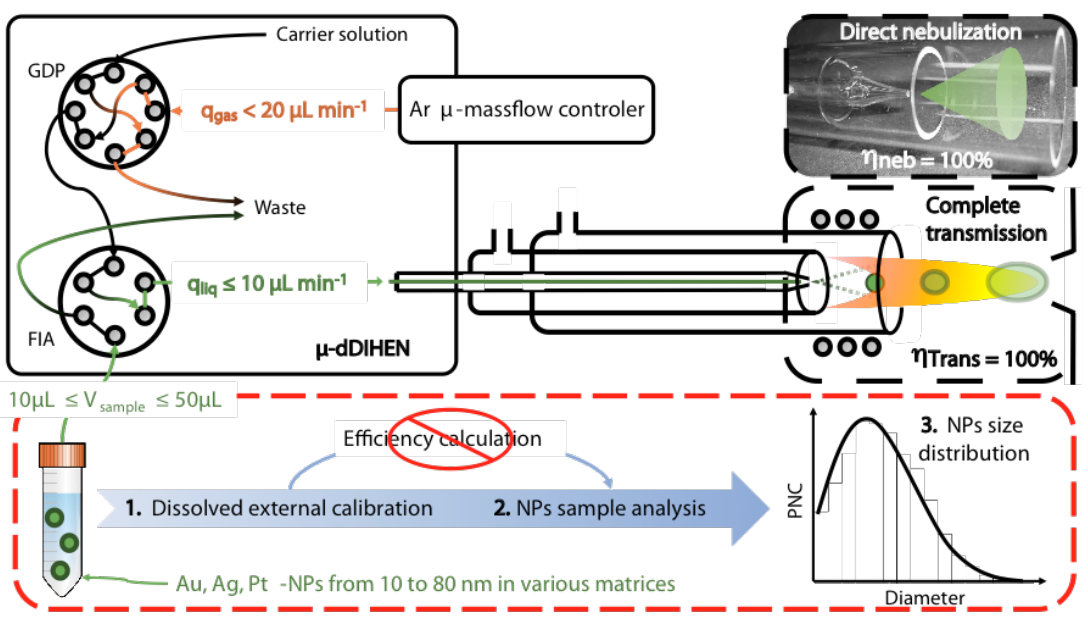

\section{Abstract}

Measurement of nanoparticle (NP) concentration and size by single particle ICP-MS (spICP-MS) usually requires the use of a NP reference material to determine the loss of NPs and/or ions during their transport from the sample solution to the detection system. The determination of this loss, qualified as nebulization efficiency $\left(\eta_{\text {Nebulization }}\right)$ and/or transport efficiency ( $\eta_{\text {Transport }}$ ), is time-consuming, costly and lacks reliability. Nebulization of the NPs directly into the plasma (without a spray chamber) results in $\eta_{\text {Nebulization }}=100 \%$ and is thus a promising strategy to avoid these calibration steps. In this work, we used the $\mu$-dDIHEN introduction system: a demountable direct injection high efficiency nebulizer (dDIHEN) hyphenated to a flow-injection valve and a gas displacement pump. For the first time with a continuous flow nebulizer, complete transport efficiency was reached (i.e. $\eta_{\text {Transport }}$ $=100 \%$ ). Operated at very low uptake rate (as low as $8 \mu \mathrm{L} \mathrm{min}^{-1}$ ), the $\mu$-dDIHEN accurately and reproducibly determined average diameters of Au-, Ag- and Pt-NPs, in full agreement with their reference values. It was also successfully tested for Au-NPs in complex matrices, such as surface waters. spICP-MS analyses with the $\mu$ - 
dDIHEN sample introduction system only require a dissolved standard calibration to determine NP average diameter $\left(d_{N P S}\right.$ in $\left.\mathrm{nm}\right)$ and number concentration $\left(N_{N P S}\right)$ from the simplified set of equations:

$$
\overline{d_{N P S}}=\sqrt[3]{\frac{6 \cdot\left(\overline{I_{N P S}}-I_{b c k g d}\right) \cdot t_{d} \cdot q_{l i q}}{S \cdot f_{a} \cdot \pi \cdot \rho}} \quad \text { and } \quad \overline{N_{N P S}}=\frac{\overline{D_{N P S}}}{q_{l i q} \cdot D \cdot t_{d}}
$$

\section{Keywords}

spICP-MS, $\mu$-dDIHEN, direct injection, complete transport efficiency, micro-uptake rate, Au- Ag- Pt-NPs.

\section{Acknowledgments}

The authors would like to acknowledge the support by IPGP multidisciplinary program PARI and by Region île-de-France SESAME Grant no. 12015908. This study contributes to the IdEx Université de Paris ANR18-IDEX-0001. Special thanks to James Ranville and Robert Reed for their constructive comments, which greatly helped to improve the quality of the manuscript and Supplementary Information.

\section{Introduction}

In addition to nanoparticles (NPs) that are naturally present (i.e. volcanic ashes, clays, magnetotactic bacteria...) and accidentally produced by human activities (i.e. engine exhausts, mining...), NPs are also increasingly manufactured for targeted applications such as cosmetics and medicine. As a consequence, increasing NP spread in the environment is becoming a growing health concern and methodologies for characterizing their origin, tracking their fate and understanding their behaviour in various media (i.e. water, soils, biological fluids...) need to be developed. Classical NP analysis techniques (i.e. Dynamic Light Scattering, Transmission Electron Microscopy...) still suffer from matrix effects, slowness, large sample volume consumption or high detection limits that hamper a reliable characterization and/or quantification of the NPs in complex media [1, 2]. Since its introduction by Degueldre et al (2005) [3], single-particle inductively coupled plasma mass spectrometry (spICP-MS) has emerged as a sensitive and robust method to characterize NPs and is becoming a reference technique for the direct determination of the size and the number of inorganic (i.e. metallic) nanoparticles. The mono- or multi-elemental composition of NPs (i.e. depending on the ICP-MS used) can be

54 determined in aqueous solutions at low environmental concentrations $\left(<10^{6}\right.$ particles $\left.\mathrm{mL}^{-1}\right)$ with the need of only limited pre-treatment of the sample (i.e. dilution) [4,5]. The spICP-MS technique requires that the loss of material occurring between the sample introduction and the detection system be determined. The so-called "transport efficiency" $\left(\eta_{\text {Transport }}\right)[6]$ accounts for material losses occurring at different places during NP transport in the ICP- 
MS, as compared to those from a dissolved standard solution of the targeted element. This transport efficiency is commonly determined with a gold NP reference material of known particle size and concentration and applied to the unknown NP sample analyses to determine their NP size and number concentrations, assuming identically behaviour of all NPs in the spray chamber and ICP-MS. Though, depending on the NP size, composition or media in which NPs are dispersed, the transport efficiency can also change. All these assumptions can de facto lead to discrepancies between the certified/true values and experimental results [7].

In the literature $[8,9], \eta_{\text {Transport }}$ between 1 and $10 \%$ is usually reported for spICP-MS with conventional concentric nebulizers at uptake rates ranging from 0.1 to $1 \mathrm{~mL} \mathrm{~min}^{-1}$ : most of the liquid sample flows to waste from the cyclonic or double-pass spray chambers. Reaching higher $\eta_{\text {Transport, }}$ and ideally $100 \%$ (i.e. complete transport efficiency), would improve the reliability of NP measurements, reduce the analytical costs, and allow the self-reliance of the method, by removing the $\eta_{\text {Transport }}$ calculation which requires a reference standard. Improvement in the introduction of the sample is the key to this problem as $\eta_{\text {Transport }}$ is function of the ratio of the sensitivity on the uptake rate (Equation 1 in the next section). Although decreasing the uptake rate would increase the ratio, the sensitivity would be negatively affected, as the number of ions reaching the detector is lower, preventing complete transport efficiency. Higher $\eta_{\text {Transport }}$ is, thus, the result of a low uptake rate applied to a high sensitivity introduction system. Franze et al. (2012) [10], with a three-stage Peltier cooled desolvation system (APEX Q) and a low uptake rate (i.e. $0.1 \mathrm{~mL} \mathrm{~min}^{-1}$ ), obtained $\eta_{\text {Transport }} \geq 90 \%$, the aerosol being "dried" before its introduction into the plasma. A miniaturized single-pass spray chamber, such as "total consumption (TC) sample introduction system" (originally called "Torch Integrated Sample Introduction System" - TISIS [11]), also led to a significant improvement of the transport efficiency ( $\eta_{\text {Transport }} \approx 93 \%$ ), with sample uptake rates $\leq 10 \mu \mathrm{L} \mathrm{min}^{-1}$ [12]. At such low uptake rates the evaporation process is boosted and the probability of droplet-droplet collisions is reduced [7]. Despite these important improvements, the gap to complete transport efficiency is still not filled, as droplets are still sprayed into a chamber with inevitable losses, and the $\eta_{\text {Transport }}$ calculation cannot be fully avoided. Lately, Lin et al. (2019) [13], combining APEX and the TC introduction system, both with a highperformance concentric nebulizer, claimed to reach complete transport efficiency for platinum NPs. However, in their study, the calibration was not performed using dissolved standard solutions but with nanoparticle reference materials of known size, distorting the calculated $\eta_{\text {Transport. }}$ Although it is an improvement, such a calibration requires reference materials of known sizes, and of composition identical to the NPs dispersed in the samples (i.e. 
injection systems, for which, by construction, the nebulization efficiency is $100 \%$, as the sample containing NPs is directly nebulized in the plasma (i.e. without a spray chamber).

The micro Droplet Generator $(\mu \mathrm{DG})[10,14]$ is one such direct injection techniques. It consists of a piezoelectric head dispensing fine droplets containing the NPs in a flux of helium toward the plasma. Each droplet is subjected to the same fate in the plasma as the aerosol produced with a conventional introduction system. The mentioned main advantages are that it does not require a reference material to determine the transport efficiency and that it consumes very small amounts of sample (e.g. droplet volume $c a .0 .1 \mathrm{~nL})$ [15]. However, the analysis throughput is limited, as the system is not connected to an autosampler [10]. To circumvent this drawback, Mehrabi et al. (2019) [16] recently coupled the $\mu \mathrm{DG}$ to a spray chamber. Differences between the nebulized aerosols and NP containing $\mu$-droplets were nonetheless still observed and hampered the reliability of the measurements. This is a good example that direct and total nebulization is not a guarantee of complete transport efficiency.

Direct injection nebulizers (DIN) also allow a complete nebulization of the sample into the plasma as they are directly fitted into the torch in place of the injector [17]. Such pneumatic concentric nebulizers were previously developed for low uptake rate analysis of complex matrices (i.e. biological or petroleum samples) [18, 19]. At the Institut de physique du globe de Paris (IPGP), the demountable Direct Injection High Efficiency Nebulizer (dDIHEN) [20] is commonly used for high-throughput Multi-Collection (MC)-ICP-MS measurements of boron isotope ratios in geological samples [21,22]. Compared to other DINs, the dDIHEN presents several advantages such as smaller void volume leading to lower detection limits, but, more importantly, higher sensitivity and enhanced signal stability [23]. This is because the dDIHEN can be tuned to obtain a fine droplet distribution [20], leading to improved transport and ionization of the sample in the plasma. Moreover, when a DIN is clogged or melted, the entire nebulizer has to be replaced, whereas the capillary or needle of the dDIHEN can be dismounted and replaced at low cost. Our newly developed direct injection system, the " $\mu$-dDIHEN" [24], combines the automated dDIHEN [22] to a gas displacement pump and allows spICP-MS micro-analysis at stable micro-uptake rates down to $8 \mu \mathrm{L} \mathrm{min}^{-1}$, without a spray chamber. By design, DINs have a nebulization efficiency

111 of $100 \%$, since the whole aerosol is nebulized directly in the plasma. However, in preliminary tests [24], despite 112 complete nebulization, the transport efficiency only reached about $85 \%$ for $40 \mathrm{~nm}$ Au-NPs in ultrapure water. This 113 again demonstrates that direct nebulization does not ensure complete transport efficiency. Consequently, the 114 question of whether or not complete transport efficiency can be achieved with a direct introduction system remains 115 open. In the present paper we used the $\mu$-dDIHEN, a fully automatized low-uptake rate direct injection system, on 116 a sector field inductively coupled plasma mass spectrometer (SF-ICP-MS) at different uptake rates. We first 
117 investigate transport efficiencies at variable uptake rate with a 40nm Au-NPs reference solution. Then we

118 demonstrate the unique analytical capacities of the $\mu$-dDIHEN, and the effectiveness of the optimized analytical

119 method to NPs of different sizes (i.e. 10 to $80 \mathrm{~nm}$ ) and compositions (i.e. Au-, Ag- and Pt-NPs) in various complex

120 matrices (i.e. synthetic environmental samples and a river water).

121

Experimental section

\section{Chemicals}

Ultrapure water (resistivity $\approx 18.2 \mathrm{M} \Omega \mathrm{cm}^{-1}$ ) from a Millipore Integral 5 (Millipore, USA) was used throughout the work for dilutions. Single-element standards (Au, Ag and Pt) at $1000 \mathrm{mg} \mathrm{L}^{-1}$ were obtained from

126 SCP Science (Canada). Normapure nitric and hydrochloric acid (VWR, USA) were purified in a sub-boiler DST 1000 (Savillex, USA). Au-NP reference materials with a nominal diameter of $c a .10,15,20,40,60$ and $80 \mathrm{~nm}$

128 were obtained from British Biocell International (UK). Ag-NP reference materials with a nominal diameter of $c a$. 20, 30 and $40 \mathrm{~nm}$ as well as $c a .50 \mathrm{~nm}$ Pt-NP reference material were obtained from nanoComposix (USA). All

130 NPs are spherical and citrate capped. Average diameters and particle number concentrations provided by suppliers on the certificate of analysis (CofA) are listed in Table 1. The different NP solutions were sonicated in a bath (60

132 seconds at $42 \mathrm{kHz}$ ) and freshly diluted prior to spICP-MS analysis. Concentrations were adapted for each flowrate to avoid coincidences of NP occurrence at millisecond dwell-time.

134 Table 1 Reference and experimental average diameters ( $\mathrm{nm})$ and associated standard deviation $(\mathrm{n}=5)$ of $\mathrm{Au}$, Ag and 135 Pt-NPs. Experimental determination was done with the $\mu$-dDIHEN at $8 \mu \mathrm{L} \mathrm{min}{ }^{-1}$

\begin{tabular}{lcc|cc} 
& Ref. diameter $(\mathbf{n m})$ & Ref. $S D(n m)$ & Exp. diameter $(\mathbf{n m})$ & Exp. SD (nm) \\
\hline Au-NPs & 10.0 & 1.0 & 9.7 & 0.3 \\
& 15.0 & 1.5 & 17.5 & 0.4 \\
& 20.3 & 1.6 & 21.0 & 0.3 \\
& 40.8 & 3.3 & 40.7 & 0.2 \\
& 60.8 & 4.9 & 53.5 & 0.1 \\
\hline Ag-NPs & 79.8 & 6.4 & 59.5 & 0.4 \\
& 19.7 & 3.6 & 23.1 & 0.3 \\
& 31.0 & 3.0 & 29.0 & 0.2 \\
\hline Pt-NPs & 41.0 & 5.0 & 40.0 & 0.4
\end{tabular}


tuned to ensure maximum sensitivity and stability, and low uranium oxide formation ( $\mathrm{UO} / \mathrm{U} \leq 7 \%$ ). Also, trapezium-like peak shape was verified at each flow-rate using a $1000 \mathrm{ng} \mathrm{L}^{-1}$ dissolved gold solution. Table 2 gives for spICP-MS using the SF-ICP-MS is $1 \mathrm{~ms}$ since the magnet needs to stabilize between two measurements.

\section{$\mu-d D I H E N$ system}

$\mathrm{Ni}:$ « $\mathrm{H} »$ skimmer and sampler

Cones

Uptake rate

RF power

Ar Cool gas

Ar auxiliary gas

Ar nebulization gas

Monitored isotopes

NPs measurement

Oxides
8 to $38 \mu \mathrm{L} \mathrm{min}{ }^{-1}$

$1360 \mathrm{~W}$

\section{Conventional spray chamber}

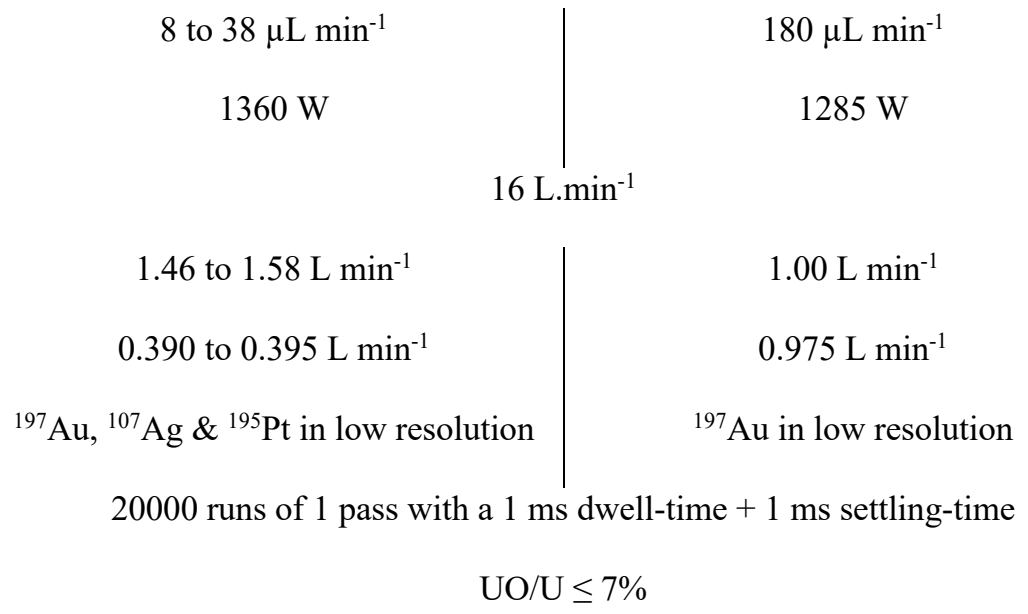

Most of the measurements were achieved using the $\mu$-dDIHEN system developed by Louvat et al. (2019).

145 It is assembled as i) a dual-loop gas displacement pump (GDP) (8-port valve, Elemental Scientific, USA) fed by 146 an argon (Ar) micro-Mass-Flow Controller $\left(17 \leq q_{A r-M C F} \leq 75 \mu \mathrm{L} \mathrm{min}{ }^{-1}\right.$, El-flow, Bronkhorst, Netherlands)

147 delivering a stable low velocity flow of carrier solution $\left(2 \% \mathrm{HNO}_{3}\right.$ or $\left.\mathrm{HCl}\right)$ to ii) a Flow-Injection Analysis valve 148 (6-port FAST valve, Elemental Scientific, USA) connected to an autosampler (SC-DX, Elemental Scientific, USA) and equipped with a micro-volume loop (of $50 \mu \mathrm{L}$ ) linked to iii) a demountable high efficiency direct injection nebulizer (dDIHEN, Analab, France) plugged in the torch, nebulizing the sample directly into the plasma. The GDP valve is toggled every 30 seconds, which is long enough to have the GDP loops filled with the carrier solution and quick enough to avoid Ar being pushed towards the nebulizer since any disruption of the liquid flow rate (Ar or air bubbles) induces the extinction of the ICP-MS plasma [21, 22]. Another program (Elemental Scientific,

154 USA) controls the filling of the sample loop from the auto-sampler on the FIA valve and synchronizes MS measurements and sample injections. All these parameters were optimized in a previous study [24]. Note that 17 $\mu \mathrm{L} \min ^{-1}$ is the lowest possible stable gas rate dispensed by the mass-flow controller of the GDP. 

solution; iii) solution ii with $100 \mathrm{mg} \mathrm{L}^{-1} \mathrm{CaCO}_{3}$ and iv) an ultra-filtered (1 $\mathrm{kDa}$ membrane pore size) Seine river water $(\mathrm{SRW})\left([\mathrm{Ca}]=123 \mathrm{mg} \mathrm{L}^{-1} ;[\mathrm{Na}]=80 \mathrm{mg} \mathrm{L}^{-1} ;[\mathrm{K}]=[\mathrm{Mg}]=16 \mathrm{mg} \mathrm{L}^{-1} ; \mathrm{DOC}=2.5 \mathrm{mg} \mathrm{L}^{-1} ; \mathrm{pH}=\right.$ 7.2: $\left.\sigma=195 \mu \mathrm{S} \mathrm{cm}^{-1}\right)$.

Additional measurements with a conventional spray chamber were performed using a $\mu$ flow-PFA nebulizer connected to a Peltier cooling $\mathrm{PC}^{3}$ system (Elemental Scientific, USA) at $20^{\circ} \mathrm{C}$. Operating parameters are listed in Table 2.

\section{Data processing}

The following steps were programmed in MATLAB (R2018b) using the raw data exported from the SFICP-MS sequence. Size distributions were calculated using the dissolved $\mathrm{Au}, \mathrm{Ag}$ and Pt standards calibration curves to calculate a mass per particle from the signal intensities of particle events, which were then converted to a size, assuming a spherical shape for the NPs.

\section{- Calculation of the transport efficiency ( $\left.\eta_{\text {Transport }}\right)$ using $40 \mathrm{~nm}$ Au-NPs}

173 is also needed, as $\eta_{\text {Transport }}$ compares the signal effectively measured for this solution to the signal that can be calculated with the dissolved Au calibration only. As the signal produced by Au-NPs is very distinct from the dissolved threshold, with and without dissolved gold, the method of the $3 \sigma$ cut-off $\left(D_{\min }\right.$, calculated as [25]) is applied for the definition of the threshold between dissolved and NP signals. Using the established spICP-MS theory (the size method from [6]) and the full demonstration given in the Supplementary Information section, $\eta_{\text {Transport }}$ is calculated as:

$$
\eta_{\text {Transport }}=\frac{d_{A u N P s}{ }^{3} \cdot S \cdot f_{a} \cdot \pi \cdot \rho}{6 \cdot\left(I_{N P s}-I_{b c k g d}\right) \cdot t_{d} \cdot q_{l i q}}
$$

Equation 1

where $d_{A u-N P S}$ is the Au-NP average diameter (in $\mathrm{nm}$, here $40.8 \mathrm{~nm}$ ), $S$ is the slope of the external dissolved calibration (in cps $\mathrm{ng}^{-1} \mathrm{~L}$ ), $f_{a}$ is the mass fraction of gold in the Au-NPs (here $f_{A u-N P S}=1$, pure Au-NPs), $\rho$ is the density of the NPs $\left(\rho_{A u-N P S}=19.32 \times 10^{-12} \mathrm{ng} \mathrm{nm}^{-3}\right), I_{N P S}$ is the average measured signal produced by Au-NPs (in cps), $I_{b c k g d}$ is the average background signal of gold (in cps), $t_{d}$ is the dwell-time (in min) and $q_{\text {liq }}$ is the liquid flowrate $\left(\right.$ in $\left.\mathrm{L} \mathrm{min}^{-1}\right)$. Note that the y-intercept of the calibration curve does not appear in Equation 1 as it is included into $I_{b c k g d}$ that contains both instrumental and dissolved ion backgrounds. 

number concentration (PNC)

We distinguish below the transport and nebulization efficiencies (respectively noted $\eta_{\text {Transport }}$ and $\left.\eta_{\text {Nebulization }}\right)$ for the calculation of the particle size and number concentration. Details about the difference between these efficiencies are provided in the "Result and discussion" section and in Supplementary Information (which includes also a demonstration of the equations).

192 By inverting Equation 1, PSD is calculated from the signal distribution of each NP suspension solution. For a 193 known chemical composition and assuming a spherical shape, the diameter of the $\mathrm{i}^{\text {th }}$ bin (with $I_{i-N P S}$ the intensity 194 of the $\mathrm{i}^{\text {th }}$ bin) is given by:

$$
d_{i-N P S}=\sqrt[3]{\frac{6 \cdot\left(I_{i-N P S}-I_{b c k g d}\right) \cdot t_{d} \cdot q_{\text {liq }} \cdot \eta_{\text {Transport }}}{S \cdot f_{a} \cdot \pi \cdot \rho}}
$$

Equation 2

Then, the average diameter can be calculated as:

$$
\overline{d_{N P S}}=\sqrt[3]{\frac{6 \cdot\left(\overline{I_{N P S}}-I_{b c k g d}\right) \cdot t_{d} \cdot q_{\text {liq }} \cdot \eta_{\text {Transport }}}{S \cdot f_{a} \cdot \pi \cdot \rho}}
$$

The number concentration for each $\mathrm{i}^{\text {th }}$ bin is :

$$
N_{i-N P s}=\frac{D_{i-N P S}}{\eta_{\text {Nebulization }} \cdot q_{\text {liq }} \cdot D \cdot t_{d}}
$$

Equation 4

And the total number concentration is:

$$
\overline{N_{N P S}}=\frac{\overline{D_{N P S}}}{\eta_{\text {Nebulization }} \cdot q_{\text {liq }} \cdot D \cdot t_{d}}
$$

where $D_{N P S}$ the total number of NP events.

\section{Results and discussion}




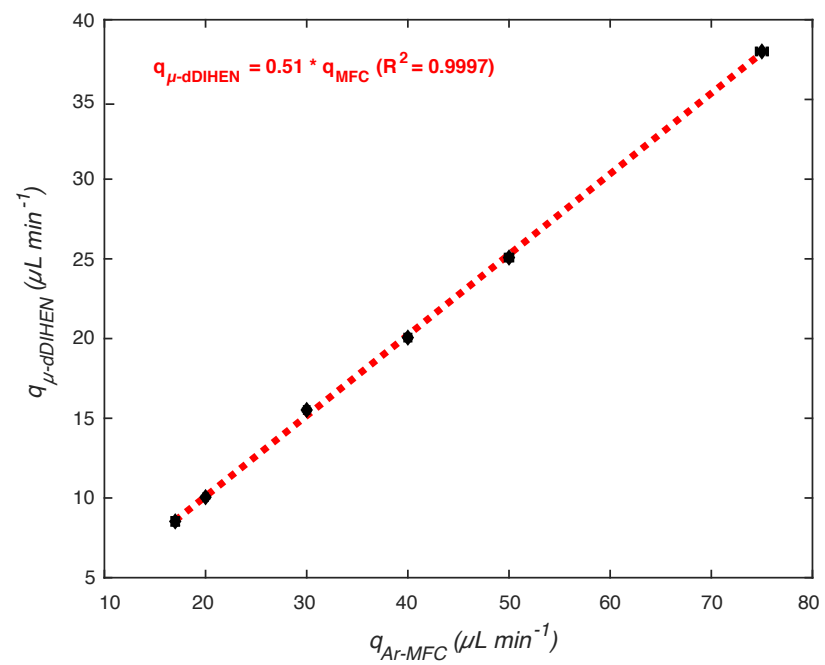

$213 \mu$-dDIHEN system uses a gas displacement pump (GDP) with a micro-mass-flow controller (MFC), which was

214 tested between 17 and $75 \mu \mathrm{L} \mathrm{min}^{-1}$ of Ar. The corresponding liquid uptake rates for the $\mu$-dDIHEN were calculated

215 by dividing the known sample loop volume (here $50 \mu \mathrm{L}$ ) by the peak duration (between the beginning of the signal

216 increase and the beginning of its decrease as defined in [24]). For the system used in this study, liquid uptake rates

217 are always half the gas flow rates (i.e. 8 to $38 \mu \mathrm{L} \mathrm{min}{ }^{-1}$, respectively, Fig. 1). This difference between gas flow

218 rates and liquid uptake rates is due to the small inner diameter of the capillaries used (i.e. few hundreds of $\mu \mathrm{m}$ )

219 and, more importantly, to the strong counter-pressure at the tip of the nebulizer generated by the large difference

220 of surface area between the entrance and the outlet of the nebulizer [26] (the nebulizer needle inner diameter goes

221 from $5 \mathrm{~mm}$ to $0.3 \mathrm{~mm}$ ). Moreover, the compressible gas partly absorbs the liquid pressure after the mass flow

222 controller, reducing its volume, thus slowing the flow. The $\mu$-dDIHEN system needs about one hour at the

223 beginning of the session (i.e. after plasma ignition) to stabilize pressure and flow [24].

Influence of the uptake rate on gold dissolved signal

Fig. $2 \mu$-dDIHEN liquid uptake $\left(q_{\mu-d D I H E N}\right)$ influence on a) dissolved ${ }^{197} \mathrm{Au}$ sensitivity $\left.\left(S_{197 A u}\right), \mathrm{b}\right)$ Au-NPs intensity $\left(I_{A u N P S}\right)$ and c) transport efficiency $\left(\eta_{\text {Transport }}\right) .{ }^{197} \mathrm{Au}$ sensitivity is the slope of the dissolved Au calibration at each liquid uptake.

227 Dashed red lines represent a) linear fit from the least square method, b) constant intensity and c) complete nebulization 
efficiency. Dashed black arrows represent sensitivity decrease factor $\beta$ for a) dissolved Au and b) Au-NP. Green stars are data previously published in [24]
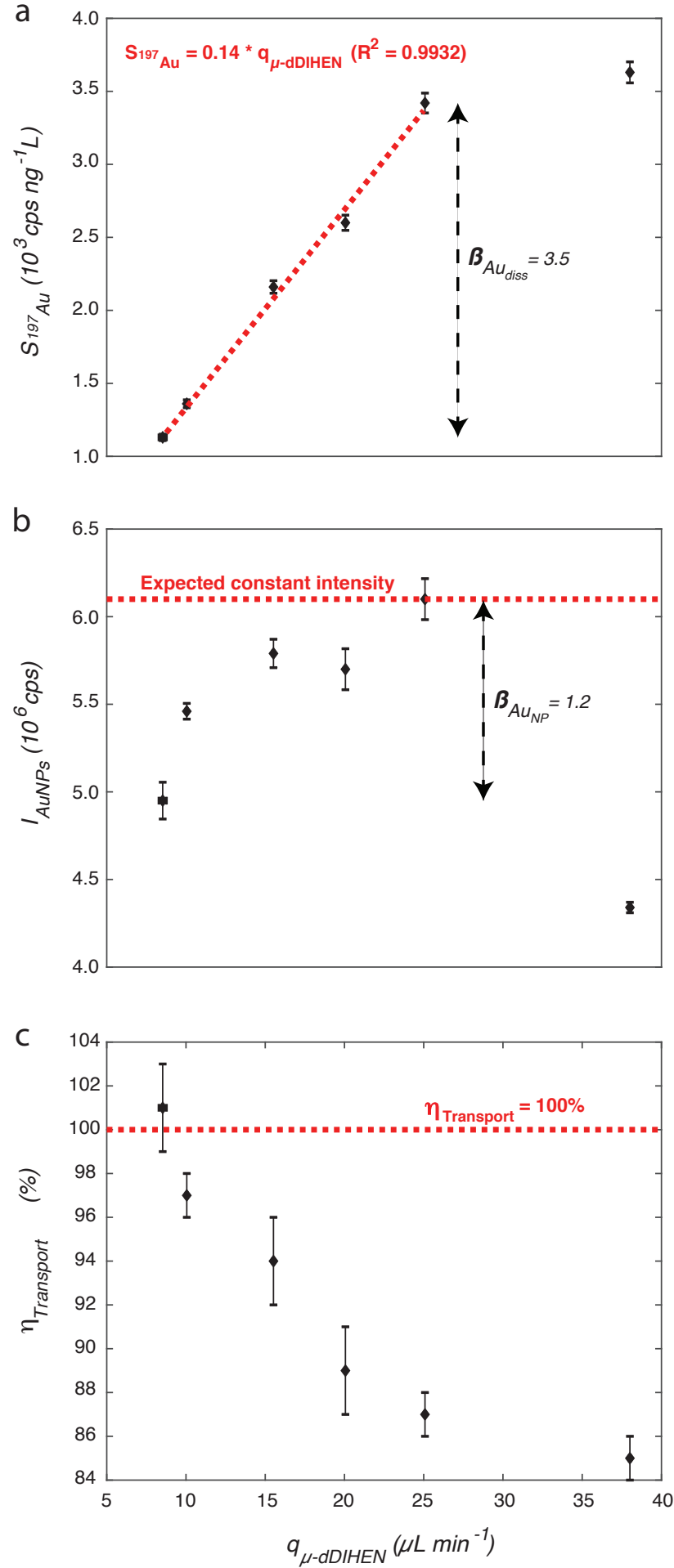

Within the gas flow range 17-75 $\mu \mathrm{L} \mathrm{min}^{-1}$ the signal sensitivity for dissolved gold was measured at six

232 different rates $\left(17,20,30,40,50\right.$ and $\left.75 \mu \mathrm{L} \mathrm{min}^{-1}\right)$, sensitivity being the slope of an external calibration curve with

2336 solutions of known [Au] between 10 and $10000 \mathrm{ng} \mathrm{L}^{-1}$. At each flow rate, the coefficient of determination $-\mathrm{R}^{2}$

234 - was calculated to verify the linearity of the external calibration and no significant variation was observed with

235 flow rate (i.e. $\left.\mathrm{R}^{2}=0.9989+/-0.0005\right)$. However, as previously observed for ${ }^{11} \mathrm{~B}$ or ${ }^{238} \mathrm{U}[24]$, the ${ }^{197} \mathrm{Au}$ sensitivity 
236 linearly increases with the uptake rate between 8 and $25 \mu \mathrm{L} \mathrm{min}^{-1}$, as more atoms are introduced per unit of time, 237 until $25 \mu \mathrm{L} \mathrm{min}{ }^{-1}$ (Fig. 2a) and then it plateaus beyond $25 \mu \mathrm{L} \mathrm{min}^{-1}$. This is because too high of a liquid to gas ratio 238 lowers the quality of the aerosol by spreading larger and polydisperse droplets, with heterogeneous velocities, 239 leading to extra-wet conditions that hamper a correct ionisation of the sample [27]. Increasing the nebulization gas 240 flow-rate would allow smaller droplets to be produced, even at high liquid uptake rates, but would reduce the 241 droplet residence time within the plasma, and thus the sensitivity. Ideally, the generated aerosol should be 242 composed of small and monodisperse droplets with uniform (and not too high) velocities to improve their 243 ionization. Note that in this study, for each liquid uptake rate, the nebulization gas flow-rate was carefully 244 optimized for highest sensitivity and best stability of the ICP-MS signal. The variation in nebulization gas flow245 rate was however negligible $(0.390$ to $0.395 \mu \mathrm{L} \mathrm{min}-1$, Table 2). Because of the good correlation between 246 sensitivities and uptake rates between 8 and $25 \mu \mathrm{L} \mathrm{min}^{-1}$ (Fig. 2a), we can state that the size and velocity 247 distributions of the $\mu$-dDIHEN aerosol are stable over this range of uptake rates.

For $40 \mathrm{~nm}$ Au-NPs, the sensitivity drops for uptake rates exceeding $25 \mu \mathrm{L} \mathrm{min}{ }^{-1}$ (Fig. 2b), because the water content is too high and droplet velocity is heterogeneous, preventing a full ionization of the NPs, as was observed for dissolved gold. Compared to dissolved gold, varying the uptake rate between 8 and $25 \mu \mathrm{L} \min ^{-1}$ should not affect the sensitivity for the Au-NPs: the number of Au atoms (and thus ions) being constant for a given

253 NP size, the average intensity produced by the NP spikes should be constant. Contrary to this, we observe a 254 decrease in the NP Au signal when the uptake rate decreases. This is due to longer residence times of the NPs in 255 the plasma at lower uptake rates [28]. The sampling of the ion beam by the cones occurs further from the point 256 where a single NP is completely vaporized/ionized. Due to the diffusion of the ions produced by a single NP as 257 they travel through the plasma, the ion beam width (in time) is larger when the uptake rate decreases, and its signal, 258 sampled by the cones, is smaller (in intensity). Increasing the nebulization gas flow-rate would probably shorten 259 the ion beam residence time in the plasma and increase the NP signal. However, Bings et al. (2014) [26] mentioned 260 that increasing the nebulization gas affects the stability of the signal, as it conflicts with the plasma channel gas. 261 To further investigate these competing effects, it would be interesting to operate spICP-MS at microsecond dwell262 times in order to decompose the signal of a single NP spike and calculate its peak area and duration. The produced 263 ion cloud should last longer at smaller uptake rates [28]. However, it is technically difficult to run microsecond 264 dwell-times with the SF-ICP-MS Element II, since it was not designed for fast-spICP-MS. 
Interestingly, the difference in sensitivity between dissolved ions and NPs increases with decreasing uptake rates (i.e. from 38 to $8 \mu \mathrm{L} \mathrm{min}^{-1}$ ): the dissolved gold signal drops by a factor 3.5 ( $\beta_{\text {diss- }}$, , Fig. 2a), when the AuNP signal only decreases by a factor $1.2\left(\beta_{A u-N P}\right.$, Fig. $\left.2 \mathrm{~b}\right)$. Ultimately, this allows for better discrimination of the NP signal from the dissolved signal at lower uptake rates, which can be very valuable for the characterisation of NPs in matrices with high dissolved background (i.e. geological, biological, environmental samples) since the detection level of NPs $\left(D_{\min }\right)$ is lowered.

As shown in the introduction and in the Supplementary Information, the transport efficiency ( $\left.\eta_{\text {Transport }}\right)$ designates the efficiency with which an atom coming from a NP is transported from the solution to the detection system relative to an atom coming from a dissolved sample. $\eta_{\text {Transport }}$ calculated for the $40 \mathrm{~nm}$ Au-NP solution at the different uptake rates, based on Equation 1, increases from $87 \pm 1 \%$ to $101 \pm 2 \%(n=5)$ for uptake rate decreasing from 38 to $8 \mu \mathrm{L} \mathrm{min}{ }^{-1}$ (Fig. 2c). Thus, as previously shown in a preliminary study [24], despite a nebulization efficiency of $100 \%$ using a direct injection system, $\eta_{\text {Transport }}$ can be incomplete. As shown in the Supplementary Information and in Equation 6, the transport efficiency covers the material losses in the introduction system ( $\left.\eta_{\text {Nebulization }}\right)$ and the losses in the ICP-MS for NPs relative to dissolved sample $\left(\alpha_{\text {Transmission }}\right)$ :

$$
\eta_{\text {Transport }}=\eta_{\text {Nebulization }} \cdot \alpha_{\text {Transmission }}
$$

Equation 6 a NP do not behave identically in the ICP-MS and that during the transport of NPs towards the detection system downstream to the nebulization, other phenomena occur. Particularly, the density of ions coming from solute or from NPs might be different, creating different ion transit bias effects. Also, the ion flux to the detection system is continuous for solute while it is discrete for NPs. Altogether, this allows us to affirm that transport and nebulization efficiencies must be considered independently for the calculation of the NP size/mass and of the particle number concentration, respectively.

We demonstrate here that $\alpha_{\text {Transmission }}=1$ can be achieved using a direct injection nebulizer with continuous

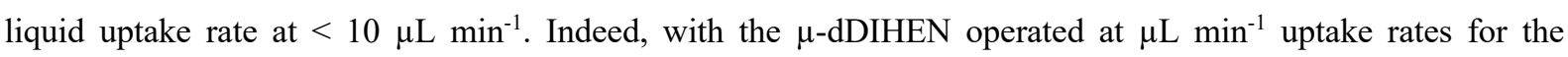
measurement of $40 \mathrm{~nm}$ Au-NPs, we reached, for the first time, both $\eta_{\text {Nebulization }}=100 \%$ and $\eta_{\text {Transport }}=100 \%$.

292 Although, as described in the introduction, other studies have shown potential improvements towards complete transport efficiency, introduction systems are still nebulizing through a spray chamber into the plasma leading to 
(i.e. multiple elements within the same NP). For example, concerning the most recent $\mu$ DG methodology [16], the vaporization/ionization differences observed between the aerosol nebulized from a spray chamber and the $\mu$ droplets from the $\mu \mathrm{DG}$ system are, basically, similar to the incomplete transport efficiency we witnessed here for uptake rates exceeding $10 \mu \mathrm{L} \mathrm{min}^{-1}$. Consequently, in all above studies the calculation of the transport efficiency is still mandatory for spICP-MS analysis. The use of the $\mu$-dDIHEN at its lowest uptake rate (i.e. $\left.8 \mu \mathrm{L} \mathrm{min}^{-1}\right)$ does not require calculation of the nebulization or the transport efficiency, as $\eta_{\text {Nebulization }}$ and $\eta_{\text {Transport }}$ are equal to $100 \%$. Thereby, the $\mu$-dDIHEN system, operated below $10 \mu \mathrm{L} \min ^{-1}$ makes spICP-MS analysis much more straightforward. Note that the increase of $\eta_{\text {Transport }}$ also leads to a noticeable decrease of the minimum detectable NP diameter $D_{\min }$ from $8 \mathrm{~nm}$ at $38 \mu \mathrm{L} \mathrm{min}-1$ to $6.5 \mathrm{~nm}$ at $8 \mu \mathrm{L} \mathrm{min}^{-1}$. This is mainly due to the fact that the signal from the blank, and consequently its $3 \sigma$ used for the calculation [25], is decreasing with the flow-rate.

To further validate the $\mu$-dDIHEN measurement method, we tested if complete transport was achieved independently of the NP size or composition, and if measurements were reproducible over 12 hours and between measurement sessions months apart.

\section{Measurement of Au-NP, Ag-NP and Pt-NP reference materials under optimal $\mu$-dDIHEN conditions}

Fig. 3 Analysis of Au-NP reference materials with sizes between 10 and $80 \mathrm{~nm}$ using the $\mu$-dDIHEN system. a) Nanoparticle size distributions b) experimental average sizes against reference sizes in black diamonds (left y-axis) and ratio of " $\mu$-dDIHEN sizes" and "conventional spray chamber sizes" (right y-axis). Horizontal and vertical error bars on the black diamonds represent the standard deviation of the reference material and of the triplicate measurement, respectively. Dashed red and blue lines represent the 1:1 linear fit and the average size ratio (i.e. 1.01), respectively. The blue area represents the standard deviation of the size ratio (i.e. 0.04)
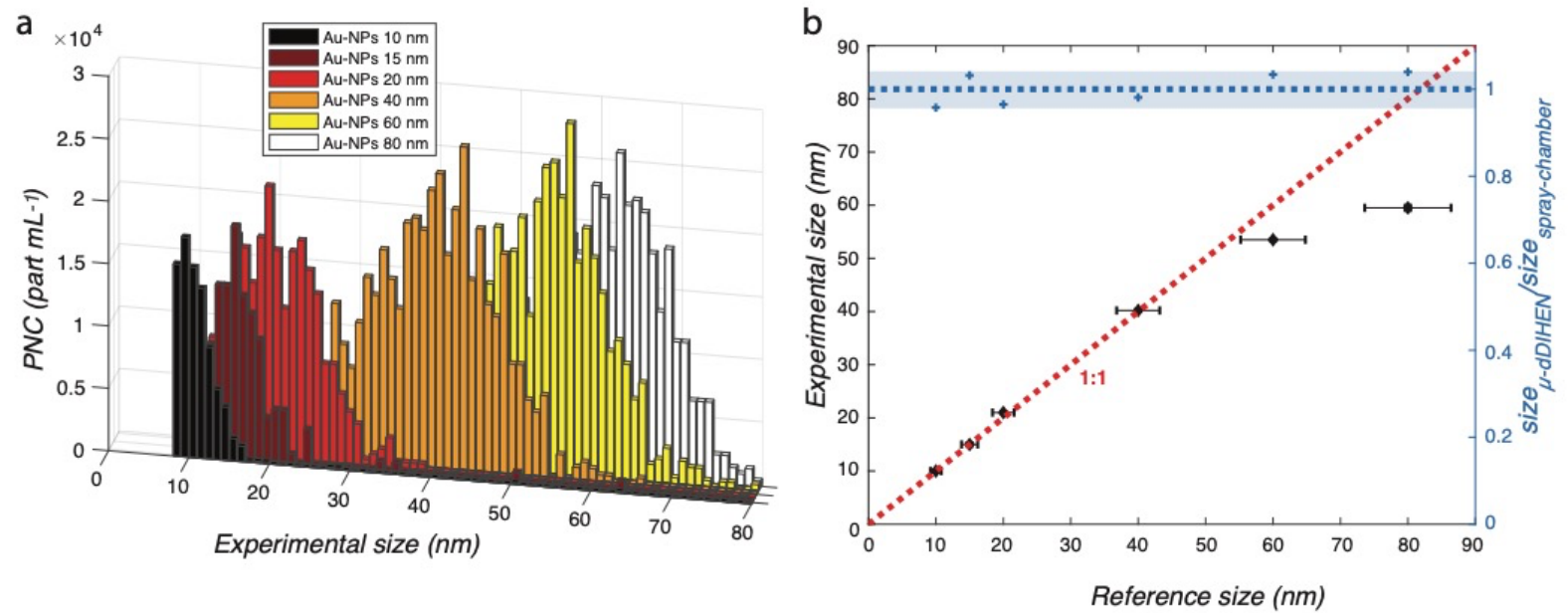

Reference Au-NPs of known sizes ( $c a$. 10, 15, 20, 40, 60 and $80 \mathrm{~nm}$ ) were analysed and their size

317 distributions were established using Equations 2 and 4 with $\eta_{\text {Transport }}$ fixed at $100 \%$ (sensitivity for ${ }^{197}$ Au that day

318 equal to $993 \pm 16 \mathrm{cps} \mathrm{ng}^{-1} \mathrm{~L}, \mathrm{R}^{2}=0.9992$ ). If $\eta_{\text {Transport }}$ is indeed $100 \%$, the measured size should be identical to the

319 reference size. The Gaussian-like shape of the measured particle size distributions (PSD) for Au-NPs between 10

320 and $80 \mathrm{~nm}$ (Fig. 3a) shows that neither the $\mu$-dDIHEN system nor the spICP-MS parameters (i.e. settling time and 
dwell-time) alter the size distributions ( $p \leq 10^{-15}$ in the Kolmogorov-Smirnov test) [29]. Moreover, the measured and reference average diameters are in good agreement (Table 1) for Au-NPs of 10, 15, 20 and $40 \mathrm{~nm}$ diameters. For larger Au-NPs (60 and $80 \mathrm{~nm}$ ), the measured average diameters are underestimated by our method (Fig. 3b).

324 The same trend was however observed with a conventional spray chamber (i.e. size $_{\mu \text {-dDIHEN }} /$ size $_{\text {spray-chamber }}=1.01$ $325 \pm 0.04, \mathrm{n}=6$, for Au-NPs of diameters between 10 and $80 \mathrm{~nm}$ ). This underestimation arises from the non-linear response of the MS detector above a given number of counts $\sec ^{-1}$ in counting mode [30] when too large a number of ions issued from a relatively large NP reaches the detector. It was clearly observed in this study since the instrument analysis report displayed intensities of 60 and $80 \mathrm{~nm}$ Au-NPs with a "S" for "skipped" meaning that the secondary electron multiplier (SEM) jumped from pulse counting to analog mode, due to a saturation in counting mode. As a consequence, the high sensitivity of SF-ICP-MS, compared to quadrupole or time-of-flight ICP-MS [31], can be a drawback for NP measurements, because the saturation point of the SEM in counting mode can be quickly reached. It is particularly the case with elements that have isotopes with high abundance (i.e. Au with $\left.\mathrm{A}_{197 \mathrm{Au}}=100 \%\right)$ and/or low first ionisation energy.

Fig. 4 Nanoparticle size distributions independently determined ( $\mathrm{n}=3$ ), for 20, 30 and $40 \mathrm{~nm} \mathrm{Ag-NPs}$ and $50 \mathrm{~nm}$ Pt-NPs, All distributions are displayed as boxplots where the red horizontal mark indicates the median, and the bottom and top edges of the blue box indicate the $25^{\text {th }}$ and $75^{\text {th }}$ percentiles, respectively. The black whiskers extend to the most extreme data points not considered outliers. Outliers, plotted individually using the red-cross, are values outside the $99.3 \%$ confidence interval (+/-2.7SD). Note that the number of outliers is negligible for each distribution $(<1 \%)$. The black dot and the error bar represent the reference material value and its standard deviation, respectively

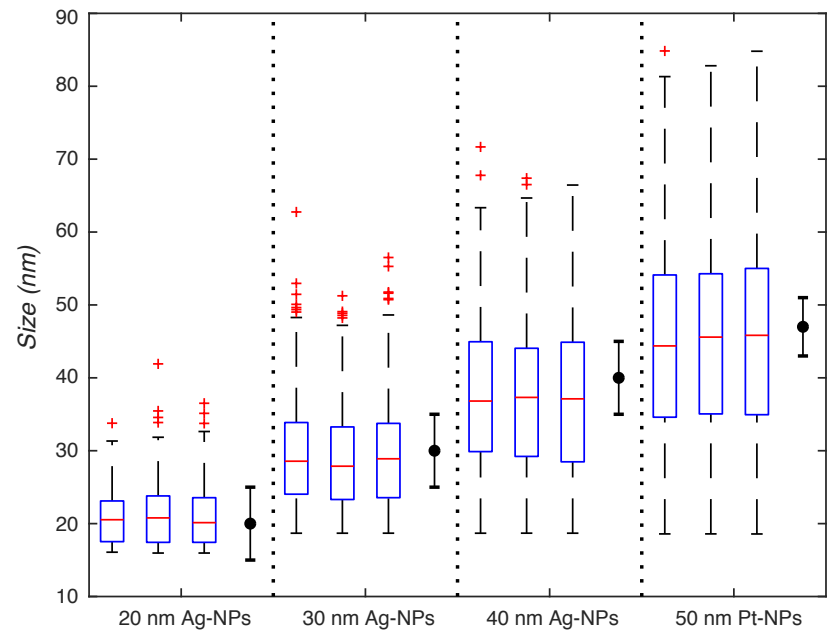

342 reference materials with the $\mu$-dDIHEN at $8 \mu \mathrm{L} \mathrm{min}{ }^{-1}$ (Fig. 4). Individual particle size distributions are displayed

343 as boxplots and were calculated using dissolved calibrations with Ag and Pt standards between 10 and $10000 \mathrm{ng}$

$344 \mathrm{~L}^{-1}\left(\mathrm{n}_{\text {standard }}=6\right)$ that gave a sensitivity of $596 \pm 2 \mathrm{cps} \mathrm{ng}^{-1} \mathrm{~L}\left(\mathrm{R}^{2}=0.9999\right)$ and $393 \pm 3 \mathrm{cps} \mathrm{ng}^{-1} \mathrm{~L}\left(\mathrm{R}^{2}=0.9998\right)$

345 for ${ }^{107} \mathrm{Ag}$ and ${ }^{195} \mathrm{Pt}$, respectively that day. As for Au-NP measurements, $\eta_{\text {Transport }}$ was fixed at $100 \%$. The average 
black dots in Fig. 4).

348 With these measurements of reference material solutions of Au-NPs at different sizes and of Ag-NPs and Pt-NPs, and provides accurate size measurement for various metallic nanoparticles, using only a sensitivity calibration with dissolved standards of $\mathrm{Au}, \mathrm{Ag}$ and Pt.

Fig. 5 Long-term stability of NPs analysis with the $\mu$-dDIHEN over 12 hours and for measurement sessions 6 months apart. a) Nanoparticle size distributions for $40 \mathrm{~nm}$ Au-NPs displayed as boxplots. The red area represents the reference size range. b) Normalized particle number concentration $\left(\alpha_{\mathrm{t}}=[\mathrm{PNC}]_{\mu \mathrm{dDIHEN}-\mathrm{t}} /[\mathrm{PNC}]_{\text {spray-chamber, }}\right.$ where $[\mathrm{PNC}]_{\text {spray-chamber }}$ is the particle number concentration determined using a conventional introduction system). The red horizontal mark indicates the median, and the bottom and top edges of the blue box indicate the $25^{\text {th }}$ and $75^{\text {th }}$ percentiles, respectively. The black whiskers extend to the most extreme data points not considered outliers. Outliers, plotted individually using the red-cross, are values outside the $99.3 \%$ confidence interval (+/- 2.7SD). Note that the number of outliers is negligible for each distribution $(<1 \%)$. The dashed red line and the red area represent the averaged value, $\alpha_{\text {AuNPs-12hours }}=0.93$ and its associated standard deviation $\mathrm{sd}=0.06$. Other displayed data (i.e. right side of each graphs) are from the same 40 $\mathrm{nm}$ Au-NPs solution, analysed 6 months earlier
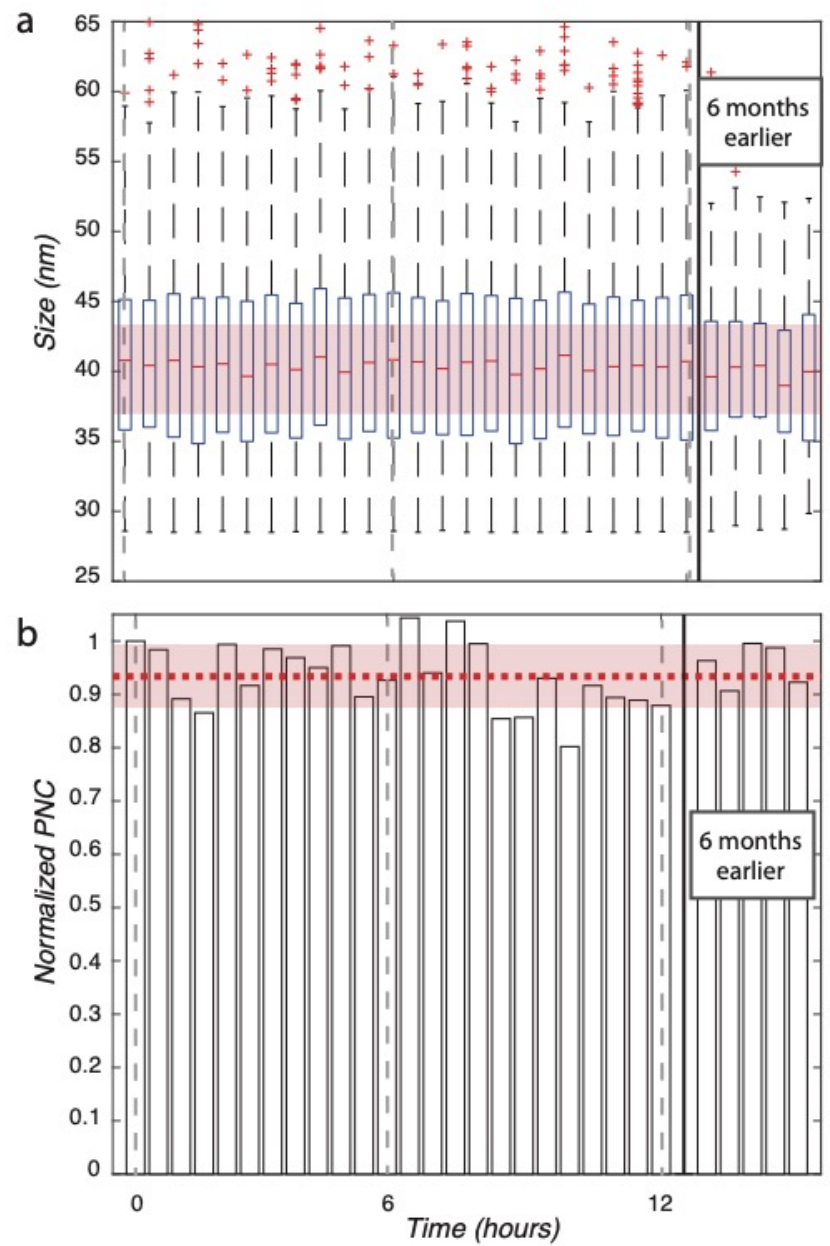

Besides the accuracy, we verified the repeatability of the $\mu$-dDIHEN spICP-MS measurements by analysing a $40 \mathrm{~nm} \mathrm{Au-NP}$ solution overnight (i.e. $\approx 12$ hours, with one measurement every 15 minutes). Boxplots of individual calculated size distributions (Fig. 5a) show that the average diameter over this relatively extended 
period of time does not vary $\left(d_{A u-N P S-12 h o u r s}=40.7 \pm 0.3 \mathrm{~nm}(\mathrm{n}=48)\right)$. Furthermore, the Gaussian-like shape of the

distribution remains identical during the analytical sequence (unchanged form of the boxes and whiskers and no increase of the number of outliers $(<1 \%)$ ). The PNC, using Equation 5 with $\eta_{\text {Nebulization }}=100 \%$ (i.e. all NPs nebulized are introduced into the plasma), was also determined over this 12-hour measurement of the Au-NP solution, and then compared to the PNC determined using a conventional introduction system (i.e. $\alpha_{t}=$ $\left.372[\mathrm{PNC}]_{\mu d D I H E N-t} /[\mathrm{PNC}]_{\text {spray-chamber }}\right)$. Fig. $5 \mathrm{~b}$ shows that the overnight reproducibility is excellent $\left(\alpha_{\text {AuNPs-12hours }}=0.93\right.$ $373 \pm 0.06(\mathrm{n}=48))$. However, after 9 hours, a decrease of the $\alpha_{A u N P s}$ by $c a .13 \%$ is observed, which is linked to an 374 instrumental drift, also regularly observed when using ICP-MS with conventional introduction system over hours. 375 As with conventional ICP-MS, the periodic measurement of a quality control (i.e. certified solution), a repeated 376 dissolved external calibration or the use of an internal standard well suited to spICP-MS [31] can rectify this drift.

377 Thereby, we can state that the prolonged use of the $\mu$-dDIHEN does not alter the measurement of the particle 378 number concentration or the average size distribution. We also measured the same Au-NP standards 6 months apart and these data of spICP-MS analysis are also displayed in both Fig. 5a and 5b. Although the PSDs appear narrower 6 months earlier, due to differences in the performance of the day (i.e. decrease of the stability), as there are no changes in the $d_{A u-N P S}$ and the $\alpha_{A u N P s}$ between the two-measurement series, we can claim that the system is robust for spICP-MS measurement even at lower NP concentration.

\section{$\mu$-dDIHEN measurement of Au-NPs in synthetic and natural matrices}

Fig. 6 Matrix testing for Au-NPs measurement with the $\mu$-dDIHEN. Nanoparticle size distributions for $40 \mathrm{~nm}$ Au-NPs displayed as boxplots in i) ultra-pure water, then spiked with ii) $5 \mathrm{mg} \mathrm{L}^{-1}$ fulvic acid (IHSS Pahokee Peat), finally additionally spiked with iii) $100 \mathrm{mg} \mathrm{L}^{-1} \mathrm{CaCO}_{3}$ and iv) in an ultra-filtered (i.e. 1 kDa membrane pore size) Seine river water (SRW). The red horizontal mark indicates the median, and the bottom and top edges of the blue box indicate the $25^{\text {th }}$ and $75^{\text {th }}$ percentiles, respectively. The black whiskers extend to the most extreme data points not considered outliers. Outliers, plotted individually using the red-cross, are values outside the 99.3\% confidence interval (+/- 2.7SD). Note that the number of outliers is negligible for each distribution $(<1 \%)$. The red area represents the reference size range

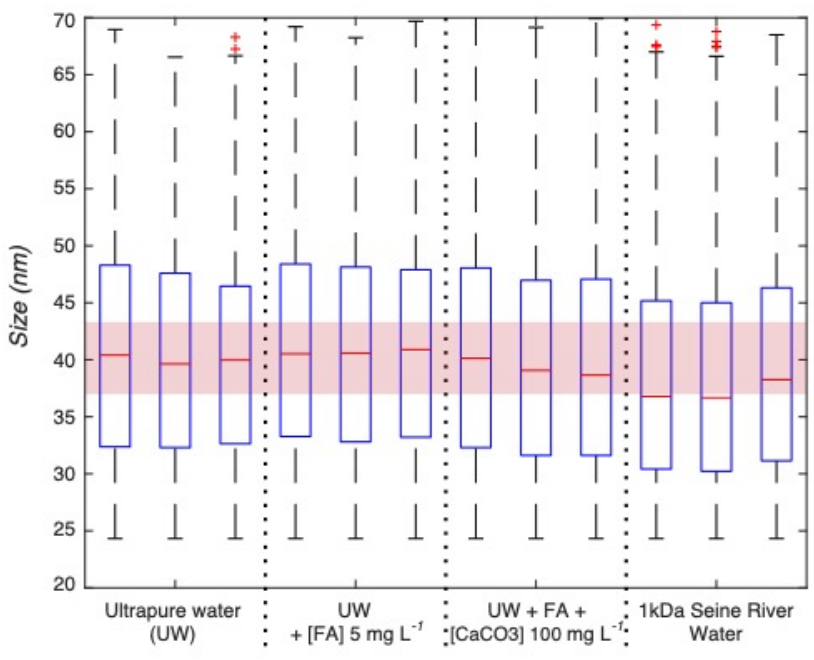


394 higher level of total dissolved solid, total organic carbon, etc), ionization of the analytes can be increased or 395 decreased as compared to ultrapure water or dilute acid, changing the sensitivity. These potential matrix effects were tested for $40 \mathrm{~nm}$ Au-NPs in three different matrices (fulvic acid, fulvic acid + calcium carbonate, and ultra-

397 filtered water from the Seine River in Paris) and compared to analysis in ultra-pure water. Individual calculated size distributions of the triplicate measurements are represented as boxplots (Fig. 6). Spiking Au-NP solution with fulvic acid and calcium carbonate has no effect on the average size and distribution spread. For the ultra-filtered Seine River water, the calculated Au-NP average size is smaller by $7.5 \%$ but with the same full width at half maximum (FWHM). This underestimation of NP size by matrix effect is not specific to the use of the $\mu$-dDIHEN, as it was reported for other introduction systems (i.e. $\mu \mathrm{DG}$ ) [14]. Matrix-matching the dissolved sensitivity calibration appears to successfully correct this underestimation [16,33], as the change in sensitivity due to the matrix is then taken into account. In our case, based on Equation 3 and the cubic-root relationship between diameter and sensitivity, the sensitivity (from dissolved calibration) should be $4.2 \%$ lower (i.e. $953 \mathrm{cps} \mathrm{ng}^{-1} \mathrm{~L}$ ) to obtain the reference Au-NP average size. Although matrix-matching calibration should be further studied to increase the accuracy of our $\mu$-dDIHEN method, the average size that was experimentally determined in the ultra-filtered Seine River water is still in reasonable agreement with the reference material value (Table 1).

\section{Conclusion}

The direct injection sample introduction system $\mu$-dDIHEN was used to directly spray solutions containing NPs into the plasma at very low uptake rate $\left(\leq 10 \mu \mathrm{L} \mathrm{min}^{-1}\right)$ and reach complete transport. The analytical

413 performance of our system was evaluated for uptake rates between 8 and $38 \mu \mathrm{L} \mathrm{min}^{-1}$. We first demonstrated that, 414 for $40 \mathrm{~nm}$ gold nanoparticles, very high transport efficiencies were always reached, and increased from $85 \%$ at 38 $415 \mu \mathrm{L} \mathrm{min}-1$ to $100 \%$ at $8 \mu \mathrm{L} \mathrm{min}^{-1}$. At this lowest uptake rate, solutions spiked with NP reference materials of different 416 size (10 to $80 \mathrm{~nm}$ ) and composition (Au-, Ag- and Pt-NPs) all showed excellent agreement between the calculated 417 and NP average sizes of the reference materials, validating the performances of our $\mu$-dDIHEN system for spICP418 MS measurements. As complete transport efficiency was reached (100\%), these results were obtained without the 419 need of a calibration with Au-NPs reference material of calibrated size; only a calibration of the sensitivity with solutions of the dissolved analyte is necessary. Environmental-like matrices (fulvic acids and calcium carbonate)

421 did not alter these performances. In water from the Seine River, although calculated size distributions were slightly smaller compared to diluted acid, these effects were not specific to the use of the $\mu$-dDIHEN and could easily be 
corrected by matrix-matching the dissolved standards used for the sensitivity calibration. Long-term repeatability over 12 hours and between measurement series 6-months apart was also achieved, showing that long series of automated analyses are possible with the $\mu$-dDIHEN. Moreover, a very high analysis throughput (i.e. 1 sample every 40 seconds in this study; total amount depending on the autosampler capacity) with very low sample consumption (the sample injection loop volume was $50 \mu \mathrm{L}$ ) is possible since the $\mu$-dDIHEN system is fully automated.

Direct nebulization, and the resulting complete transport efficiency, was thus achieved for the first time for continuous nebulization in spICP-MS, using the $\mu$-dDIHEN at $\mu \mathrm{L} \mathrm{min}{ }^{-1}$ uptake rate. In addition, we have shown that in most cases, the transmission efficiency differs between ions from solute and ions from NPs. Thus, we establish an updated calculation methodology, which distinguishes transport efficiency from nebulization 433 efficiency during the transfer of NPs from the solution to the ICP-MS detection and can be reliably used to calculate sizes and particle number concentrations. Future work will focus on the reliability of the $\mu$-dDIHEN for the determination of PNC, as this is a very important parameter. However, few Certified Reference Material with certified PNC and uncertainty are available. A step forward would also be to monitor the uptake rate on-line using a dedicated device, which would allow to more accurately determine size and PNC as well as their uncertainties using uncertainty propagation. Another way to remove the uptake rate measurement would be to use the methodology developed by Lamsal et al. (2016 - 2020) [34-35] for flow-injection analysis, which is well adapted to the $\mu$-dDIHEN system.

Additionally, a cell suspension can be nebulized into the ICP-MS for the measurement of metal content in individual cells by single-cell ICP-MS (scICP-MS). However, these cells are usually dispersed in complex 443 biological media, very fragile and/or contained in small sample volume. Nebulization through a spray chamber can lead to inaccuracy in the results because of matrix effects or cell degradation. All the benefits of the $\mu$-dDIHEN system indicate that it is well adapted to: i) monitor the metal content within individual cells for either dissolved or nanoparticulate form, ii) deal with lower cell concentration compared to conventional introduction system, and iii) minimize sample volume and preparation.

Furthermore, the use of very low $\mu$-uptake rate is also a major asset for the hyphenation of separation techniques such as asymmetrical-flow field-flow-fractionation (AF4), which could directly control the uptake rate of the $\mu$-dDIHEN thanks to the pump that delivers the carrier solution, in place of the mass-flow controller used 451 here. Finally, since there can be a significant dilution of the sample during separation, avoiding a spray chamber and the resulting sample loss is very attractive. 
454 [1]. Hochella MF, Mogk DW, Ranville J, Allen IC, Luther GW, Marr LC, et al. Natural, incidental, and 455 engineered nanomaterials and their impacts on the Earth system. Science. 2019;363.

456 [2]. Lopez-Serrano A, Olivas RM, Landaluze JS, Camara C. Nanoparticles: a global vision. Characterization, 457 separation, and quantification methods. Potential environmental and health impact. Anal Methods. 2014;(6):3845856.

459 [3]. Degueldre C, Favarger PY, Wold S. Gold colloid analysis by inductively coupled plasma-mass 460 spectrometry in a single particle mode. Anal Chim Acta. 2006;555:263-268.

461 [4]. Reed RB, Martin DP, Bednar AJ, Montano MD, Westerhoff P, Ranville JF. Multi-day diurnal measurements of Ti-containing nanoparticle and organic sunscreen chemical release during recreational use of a natural surface water. Environ Sci: Nano. 2017;4:69-77.

464 [5]. Gondikas AP, von der Kammer F, Reed RB, Wagner S, Ranville JF, Hofmann T. Release of TiO2 465 Nanoparticles from Sunscreens into Surface Waters: A One-Year Survey at the Old Danube Recreational Lake. Environ Sci Technol. 2014;48:5415-5422.

467 [6]. Pace HE, Rogers NJ, Jarolimek C, Coleman VA, Gray EP, Higgins CP, Ranville JF. Single Particle Inductively Coupled Plasma-Mass Spectrometry: A Performance Evaluation and Method Comparison in the Determination of Nanoparticle Size. Environ Sci Technol. 2011;83:9361-9369.

470 [7]. Montano MD, Olesik JW, Barber AG, Challis K, Ranville JF. Single Particle ICP-MS: Advances toward 471 routine analysis of nanomaterials. Anal Bioanal Chem. 2016;408:5053-5074.

472 [8]. Loeschner K, Brabrand MSJ, Sloth JJ, Larsen EH. Use of alkaline or enzymatic sample pretreatment prior 473 to characterization of gold nanoparticles in animal tissue by single-particle ICP-MS. Anal Bioanal Chem. $474 \quad 2014 ; 406: 3845-3851$.

475 [9]. Fréchette-Viens L, Hadioui M, Wilkinson KJ. Practical limitations of single particle ICP-MS in the determination of nanoparticle size distributions and dissolution: case of rare earth oxides. Talanta. 2017;163:121$477 \quad 126$.

478 [10]. Franze B, Strenge I, Engelhard C. Single particle inductively coupled plasma mass spectrometry: 479 evaluation of three different pneumatic and piezo-based sample introduction systems for the characterization of silver nanoparticles. J Anal At Spectrom. 2012;27:1074-1083.

481 [11]. Todoli JL, Mermet JM. New torch design with an in-built chamber for liquid sample analysis by ICP482 AES. J Anal At Spectrom. 2002;17:345-351.

483 [12]. Miyashita SI, Mitsuhashi H, Fujii SI, Takatsu A, Inagaki K, Fujimoto T. High transport efficiency of 
nanoparticles through a total-consumption sample introduction system and its beneficial application for particle size evaluation in single-particle ICP-MS. Anal Bioanal Chem. 2017;409:1531-1545.

486 [13]. Lin F, Miyashita S, Inagaki K, Liu Y, Hsu I. Evaluation of three different sample introduction systems for single-particle inductively coupled plasma mass spectrometry (spICP-MS) applications. J Anal At Spectrom. 2019;34:401-406.

[14]. Gschwind S, Hagendorfer H, Frick DA, Günther D. Mass Quantification of Nanoparticles by Single Droplet Calibration Using Inductively Coupled Plasma Mass Spectrometry. Anal Chem. 2013;85:5875-5883.

[15]. Shigeta K, Traub H, Panne U, Okino A, Rottmann L, Jakubowski N. Application of a micro-droplet generator for an ICP-sector field mass spectrometer - optimization and analytical characterization. J Anal At

494 [16]. Mehrabi K, Günther D, Gundlach-Graham A. Single-particle ICP-TOFMS with online microdroplet calibration for the simultaneous quantification of diverse nanoparticles in complex matrices. Environ Sci: Nano. 2019;6:3349-3358.

[17]. Wiederin DR, Smith FG, Houk RS. Direct injection nebulization for inductively coupled plasma mass spectrometry. Anal Chem. 1991;63:219-225.

[18]. Szpunar J, Bettmer J, Robert M, Chassaigne H, Cammann K, Lobinski R, Donard OFX. Validation of the

500 determination of copper and zinc in blood plasma and urine by ICP MS with cross-flow and direct injection nebulization. Talanta. 1997;44:1389-1396.

502 [19]. Kahen K, Strubinger A, Chirinos JR, Montaser A. Direct injection high efficiency nebulizer-inductively coupled plasma mass spectrometry for analysis of petroleum samples. Spectrochim Acta, Part B. 2003;58:397-

504413.

505 [20]. Westphal CS, Kahen K, Rutkowski WF, Acon BW, Montaser A. Demountable direct injection high 506 efficiency nebulizer for inductively coupled plasma mass spectrometry. Spectrochim Acta, Part B. 2004;59:353507368.

508 [21]. Louvat P, Bouchez J, Paris G. MC-ICP-MS Isotope Measurements with Direct Injection Nebulisation (d509 DIHEN): Optimisation and Application to Boron in Seawater and Carbonate Samples. Geostandards and 510 Geoanalytical Research. 2011;35:75-88.

511 [22]. Louvat P, Moureau J, Paris G, Bouchez J, Noireaux J, Gaillardet J. A fully automated direct injection 512 nebulizer (d-DIHEN) for MC-ICP-MS isotope analysis: application to boron isotope ratio measurements. J Anal At Spectrom. 2014;29:1698-1707. 
514 [23]. Todoli JL, Vanhaecke F. Liquid Sample Introduction and Electrothermal Vaporisation for ICP-MS:

515 Fundamentals and Applications. John Wiley \& Sons, Ltd. 2009; Chapter 5:182-227.

516 [24]. Louvat P, Tharaud M, Buisson M, Rollion-Bard C, Benedetti MF. $\mu$-dDIHEN: a new micro-flow liquid 517 sample introduction system for direct injection nebulization in ICP-MS. J Anal At Spectrom. 2019;341:553-1563.

518 [25]. Lee S, Bi X, Reed RB, Ranville JF, Herckes P, Westerhoff P. Nanoparticle Size Detection Limits by 519 Single Particle ICP-MS for 40 Elements. Environ Sci Technol. 2014;48:10291-10300.

$520 \quad$ [26]. Bings NH, von Niessen JOO, Schaper JN. Liquid sample introduction in inductively coupled plasma atomic emission and mass spectrometry - Critical review. Spectrochim Acta, Part B. 2014;100:14-37.

522 [27]. McLean JA, Zhang H, Montaser A. A Direct Injection High-Efficiency Nebulizer for Inductively Coupled 523 Plasma Mass Spectrometry. Anal Chem. 1998;70:1012-1020.

524 [28]. Olesik JW, Gray PJ. Considerations for measurement of individual nanoparticles or microparticles by 525 ICP-MS: determination of the number of particles and the analyte mass in each particle. J Anal At Spectrom. $526 \quad 2012 ; 27: 1143-1155$.

527 [29]. Massey FJ, The Kolmogorov-Smirnov Test for Goodness of Fit. J Amer Statist Assoc. 1951;46:68-78.

528 [30]. Lee WW, Chan WT. Calibration of single-particle inductively coupled plasma-mass spectrometry (SP529 ICP-MS). J Anal At Spectrom. 2015;30:1245-1254.

530 [31]. Jakubowski N, Moens L, Vanhaecke F. Sector field mass spectrometers in ICP-MS. Spectrochim Acta, 531 Part B. 1998;53:1739-1763.

532 [32]. El Hadri H, Petersen EJ, Winchester MR. Impact of and correction for instrument sensitivity drift on 533 nanoparticle size measurements by single-particle ICP-MS. Anal and Bioanal Chem. 2016;408:5099-5108.

534 [33]. Wang J, Alasonati E, Tharaud M, Gelabert A, Fisicaro P, Benedetti MF. Flow and fate of silver 535 nanoparticles in small French catchments under different land-uses: The first one-year study. Water Res. $5362020 ; 176: 115722$.

537 [34]. Lamsal RP, Jerkiewicz G, Beauchemin D. Flow Injection Single Particle Inductively Coupled Plasma 538 Mass Spectrometry: An Original Simple Approach for the Characterization of Metal-Based Nanoparticles. Anal 539 Chem. 2016;88:10552-10558.

540 [35]. Lamsal RP, Houache MSE, Williams A, Baranova E, Jerkiewicz G, Beauchemin D. Single particle 541 inductively coupled plasma mass spectrometry with and without flow injection for the characterization of nickel 542 nanoparticles. Anal Chim Acta. 2020;1120:67-74. 